\title{
Alkaloids, Coumarin and Cinnamic Acid Derivative from Murraya koenigi (Linn.) Spreng.
}

\author{
Faiza Tahia ${ }^{1}$, Md. Al Amin Sikder ${ }^{1}$, Mohammad Rashedul Haque ${ }^{1}$, J amil A. \\ Shilpi ${ }^{2}$, Khalijah Awang ${ }^{2}$, Md. Abdullah Al-Mansur ${ }^{3}$ and Mohammad A. Rashid ${ }^{1}$ \\ ${ }^{1}$ Phytochemical Research Laboratory, Department of Pharmaceutical Chemistry, Faculty of Pharmacy, \\ University of Dhaka, Dhaka-1000, Bangladesh \\ ${ }^{1}$ Center for Natural Products and Drug Discovery (CENAR), University of Malaya, \\ 50603 Kuala Lumpur, Malaysia \\ ${ }^{2}$ Department of Chemistry, Faculty of Science, University of Malaya, 50603 Kuala Lumpur, Malaysia \\ ${ }^{3}$ Bangladesh Council of Scientific and Industrial Research (BCSIR), Dr. Qudrat-I-Khuda Road, \\ Dhanmondi, Dhaka-1205, Bangladesh
}

Received: November 25, 2014; Accepted: January 15, 2015; Published (web): February 16, 2015

\begin{abstract}
A total of seven compounds were isolated from the methanol extract of leaves of Murraya koenigii (Linn.) Spreng. The isolated compounds were characterized as arborinine (1), ferulic acid (2), umbelliferone (3), mahanimbine (4), koenimbine (5), koenidine (6) and $O$-demethyl murrayanine (7) by extensive spectroscopic studies, including high field NMR analysis as well as co-TLC with authentic samples, whenever possible. This is the first report of occurrence of arborinine (1) and ferulic acid (2) from Murraya species.
\end{abstract}

Key words: Murraya koenigii, Rutaceae, arborinine, ferulic acid, umbelliferone, mahanimbine, koenimbine, koenidine, $O$-demethyl murrayanine

\section{INTRODUCTION}

Murraya koenigii (Linn.) Spreng. (Bengali name- Chotokamini; Family- Rutaceae) is more or less a deciduous unarmed shrub or a small tree up to 6 meters in height, widely distributed throughout Bangladesh. ${ }^{1}$ M. koenigii is a medicinal plant, various parts of which are used in diabetes, skin eruptions, poisonous bites, febrifuge and dysentery. ${ }^{2}$ It was found to possess antimicrobial, anthelmintic, antiinflammatory and hypoglecemic activities. $^{3}$ Previously isolated alkaloids from $M$. koenigii includes 3-methyl carbazole, murrayafoline $\mathrm{A}^{4}$, mahanimbine, koenimbine, koenidine ${ }^{5}$, mahanine, mahanimbicine $^{6}$, grinimbine, murrayanine, ${ }^{7}$ methoxy-carloazole-3-carboxylate, 1-hydroxy-3methyl carbazole ${ }^{8}$, murrayanol, girinimbilol ${ }^{9}$, bismurrayafoline E. ${ }^{10}$ The isolation of flavonoid quercetin-D-glucoside ${ }^{5}$ terpenoid like $\beta$ phellandrene, terpinen-4-ol ${ }^{11}$, and coumarin like

Correspondence to: Mohammad A. Rashid

Tel.: 9661900-73, Ext. 8137; Fax: 880-2-9667222

E-mail: rashidma@du.ac.bd

Dhaka Univ. J. Pharm. Sci. 14(1): 29-33, 2015 (June) heraclenol, heraclenin, isoheraclenin, imperatorin, umbelliferone, osthol has also been reported. ${ }^{12}$ Linalool, elemol, geranyl acetate, myrcene, alloocimene, $\alpha$-Terpinene, (E)- $\beta$-Ocimene and neryl acetate had been isolated as essential oil from this plant. ${ }^{13}$

As a part of our continuing studies with medicinal plants of Bangladesh ${ }^{14-18}$, we studied $M$. koenigii and we, herein, report the isolation of arborinine $(\mathbf{1})^{19}$, ferulic acid $(\mathbf{2})^{20}$, umbelliferone $(3)^{21}$, mahanimbine $(4)^{6}$, koenimbine $(5)^{5}$, koenidine $(6)^{5}$, and $O$-demethylmurrayanine $(7)^{22}$, where arborinine (1) and ferulic acid (2) are reported from M. koenigii for the first time.

\section{MATERIALS AND METHODS}

General experimental procedure. ${ }^{1} \mathrm{H}$ NMR and ${ }^{13} \mathrm{C}$ NMR spectra were acquired using Ultra Shield Bruker 400 and 100 NMR instrument, respectively using $\mathrm{CDCl}_{3}$ and the chemical shifts are reported in ppm with respect to TMS or residual non deuterated 
solvent signals. RV10 Basic (IKA, Germany) was used for rotary evaporation. High performance liquid chromatographic system (Shimadzu-UFLC prominence), equipped with an auto sampler (ModelSIL 20AC HT) and UV-Visible detector (Model-SPD 20A) was used for the analysis. The data were recorded using LC-solution software. Analytical reversed phase C-18 (ODS column, $250 \times 4.6 \mathrm{~mm}, 5$ $\mu \mathrm{m}$, Dynamix, Inc) was used for isolation.

Plant material. Leaves of $M$. koenigii were collected from University of Dhaka, Bangladesh, in April 2013. Voucher specimen for the plant has been maintained in Bangladesh National Herbarium, Dhaka, Bangladesh, for future reference. The leaves were first sun dried and then ground into a coarse powder using a grinding machine.

Extraction and isolation. The powdered leaf $(1000 \mathrm{~g})$ was soaked in $3.0 \mathrm{~L}$ methanol for 15 days and then filtered through a cotton plug followed by Whatman filter paper number 1 . The extract was concentrated with a rotary evaporator. A portion $(5 \mathrm{~g})$ of the concentrated methanol extract was fractionated by the modified Kupchan partitioning protocol ${ }^{23}$ into petroleum ether $(0.65 \mathrm{gm})$, carbon tetrachloride $(0.55$ $\mathrm{gm})$, chloroform $(0.30 \mathrm{gm})$ and aqueous (2.5 gm) soluble materials.

The petroleum ether soluble partitionate was subjected to gel permeation chromatography over lipophilic Sephadex LH-20 and a total of 15 fractions were collected. On the basis of their TLC behavior, fraction 8 and 9 were subjected to preparative thin layer chromatography (PTLC) using $1 \%$ ethyl acetate in toluene to yield ferulic acid $\left(2, R_{f}=0.78\right)$, mahanimbine $\left(4, R_{f}=0.64\right)$, koenimbine $\left(5, R_{f}=\right.$ $0.32)$, and koenidine $\left(6, R_{f}=0.45\right)$. Fraction 13 and 15 of the carbon tetrachloride soluble partitionate obtained from gel permeation chromatography was subjected to high performance liquid chromatography separation using $40 \%$ water in acetonitrile as mobile phase with the flow rate of $3 \mathrm{ml} / \mathrm{min}$ and pressure of $57 \mathrm{kgf} / \mathrm{cm}^{2}$ yielded arborinine $\left(1, \mathrm{R}_{\mathrm{t}}=7.228 \mathrm{~min}\right)$ and $O$-demethyl murrayanine $\left(7, \mathrm{R}_{\mathrm{t}}=10.828 \mathrm{~min}\right.$ ) (Figure $1)$.



Figure 1. Separation of compound $\mathbf{1}$ and $\mathbf{7}$ using High Performance Liquid Chromatography (HPLC).

The carbon tetrachloride soluble partitionate was subjected to gel permeation chromatography over lipophilic Sephadex LH-20 and a total of 15 fractions were collected. On the basis of their TLC behavior, fraction 15 was subjected to preparative thin layer 
chromatography (PTLC) using 30\% ethyl acetate in toluene to yield umbelliferone $\left(3, \mathrm{R}_{\mathrm{f}}=0.56\right)$.

\section{Properties of isolated compounds.}

Arborinine (1): colourless mass; ${ }^{1} \mathrm{H}$ NMR (400 $\left.\mathrm{MHz}, \mathrm{CDCl}_{3}\right): \delta 3.84(3 \mathrm{H}, \mathrm{s}, \mathrm{N}-10), 3.92(3 \mathrm{H}, \mathrm{s}, 2-$ $\left.\mathrm{OCH}_{3}\right), 4.01\left(3 \mathrm{H}, \mathrm{s}, 3-\mathrm{OCH}_{3}\right), 6.27(1 \mathrm{H}, \mathrm{s}, \mathrm{H}-4), 7.25$ $(1 \mathrm{H}, \mathrm{d}, J=8.5 \mathrm{~Hz}, \mathrm{H}-7), 7.50(1 \mathrm{H}, \mathrm{d}, J=8.5 \mathrm{~Hz}, \mathrm{H}-$ 5), $7.72(1 \mathrm{H}, \mathrm{d}, J=8.5 \mathrm{~Hz}, \mathrm{H}-6), 8.42(1 \mathrm{H}, \mathrm{dd}, J=$ 8.5, $2.0 \mathrm{~Hz}, \mathrm{H}-8) ;{ }^{13} \mathrm{C}$ NMR (100 MHz, $\left.\mathrm{CDCl}_{3}\right): \delta$ $34.2(\mathrm{~N}-\mathrm{Me}), 56.0\left(3-\mathrm{OCH}_{3}\right), 60.8\left(2-\mathrm{OCH}_{3}\right), 86.7$ (C-4), 105.7 (C-12), 114.6 (C-5), 120.7 (C-13), 121.5 (C-7), 126.7 (C-8), 130.2 (C-2), 133.9 (C-6), 140.4 (C-11), 141.9 (C-14), 156.3 (C-1), 159.4 (C-3), 180.8 (C-9).

Ferulic acid (2): white crystalline mass; ${ }^{1} \mathrm{H}$ NMR (600 MHz, $\mathrm{CDCl}_{3}$ ): $\delta 3.91(3 \mathrm{H}, \mathrm{s}, 2-\mathrm{OMe}), 5.82$ (1H, s, OH-1), 6.29 ( $1 \mathrm{H}, \mathrm{d}, J=15.9 \mathrm{~Hz}, \mathrm{H}-8), 6.95$ ( $1 \mathrm{H}, \mathrm{d}, J=8.2 \mathrm{~Hz}, \mathrm{H}-6), 7.04$ ( $1 \mathrm{H}, \mathrm{s}, \mathrm{H}-3), 7.08$ ( $1 \mathrm{H}$, br d, H-5), $7.60(1 \mathrm{H}, \mathrm{d}, J=16.0 \mathrm{~Hz}, \mathrm{H}-7)$.

Umbelliferone (3): white needle like mass; ${ }^{1} \mathrm{H}$ NMR (400 MHz, $\left.\mathrm{CDCl}_{3}\right): \delta 6.18(1 \mathrm{H}, \mathrm{d}, J=9.6 \mathrm{~Hz}$, H-3), $6.74(1 \mathrm{H}, \mathrm{dd}, J=8.0,2.1 \mathrm{~Hz}, \mathrm{H}-6), 6.77(1 \mathrm{H}, \mathrm{d}$, $J=2.0 \mathrm{~Hz}, \mathrm{H}-8), 7.29(1 \mathrm{H}, \mathrm{d}, J=8.8 \mathrm{~Hz}, \mathrm{H}-5), 7.61$ $(1 \mathrm{H}, \mathrm{d}, J=9.2 \mathrm{~Hz}, \mathrm{H}-4)$.

Mahanimbine (4): deep yellow gum; ${ }^{1} \mathrm{H}$ NMR $\left(400 \mathrm{MHz}, \mathrm{CDCl}_{3}\right): \delta 1.44\left(3 \mathrm{H}, \mathrm{s}, 3-\mathrm{CH}_{3}\right), 1.56(3 \mathrm{H}$, $\left.\mathrm{s}, 4{ }^{\prime}-\mathrm{CH}_{3}\right), 1.64\left(3 \mathrm{H}, \mathrm{s}, 4{ }^{`}-\mathrm{CH}_{3}\right), 1.76(2 \mathrm{H}, \mathrm{t}, J=8.0$ $\left.\mathrm{Hz}, 1^{\prime}-\mathrm{CH}_{2}\right), 2.15\left(2 \mathrm{H}, \mathrm{m}, 2^{\prime}-\mathrm{CH}_{2}\right), 2.32(3 \mathrm{H}, \mathrm{s}, 5-$ $\left.\mathrm{CH}_{3}\right), 5.10\left(1 \mathrm{H}, \mathrm{t}, J=7.0 \mathrm{~Hz}, 3^{\prime}-\mathrm{CH}_{2}\right), 5.61(1 \mathrm{H}, \mathrm{d}, J$ $=10.0 \mathrm{~Hz}, \mathrm{H}-2), 6.64(1 \mathrm{H}, \mathrm{d}, J=10.0 \mathrm{~Hz}, \mathrm{H}-1), 7.16$ $(1 \mathrm{H}, \mathrm{t}, J=8.0 \mathrm{~Hz}, \mathrm{H}-8), 7.29(1 \mathrm{H}, \mathrm{t}, J=8.0 \mathrm{~Hz}, \mathrm{H}-9)$, $7.36(1 \mathrm{H}$, br d, $J=8.0 \mathrm{~Hz}, \mathrm{H}-10), 7.65,(1 \mathrm{H}, \mathrm{s}, \mathrm{H}-6)$, 7.85 (1H, s, 11-NH), 7.89 (1H, d, $J=8.0 \mathrm{~Hz}, \mathrm{H}-7)$.

Koenimbine (5): white gummy residue; ${ }^{1} \mathrm{H}$ NMR (400 MHz, $\left.\mathrm{CDCl}_{3}\right): \delta 1.48(3 \mathrm{H}, \mathrm{s}, \mathrm{H}-5)$ ), 1.56 $(3 \mathrm{H}, \mathrm{s}, \mathrm{H}-4), 2.32\left(3 \mathrm{H}, \mathrm{s}, 3-\mathrm{CH}_{3}\right), 3.90(3 \mathrm{H}, \mathrm{s}, 6-$ $\left.\mathrm{OCH}_{3}\right), 5.69\left(1 \mathrm{H}, \mathrm{d}, J=10.0 \mathrm{~Hz}, \mathrm{H}-2{ }^{\prime}\right), 6.61(1 \mathrm{H}, \mathrm{d}$, $\left.J=10.0 \mathrm{~Hz}, \mathrm{H}-1^{\prime}\right), 6.94(1 \mathrm{H}, \mathrm{dd}, J=2.4,8.5 \mathrm{~Hz}, \mathrm{H}-$ 7), $7.28(1 \mathrm{H}, \mathrm{d}, J=8.5 \mathrm{~Hz}, \mathrm{H}-8), 7.41(1 \mathrm{H}, \mathrm{d}, J=2.4$ $\mathrm{Hz}, \mathrm{H}-5), 7.62$ (1H, s, H-4).

Koenidine (6): yellow gum; ${ }^{1} \mathrm{H}$ NMR (400 $\left.\mathrm{MHz}, \mathrm{CDCl}_{3}\right): \delta 1.45\left(3 \mathrm{H}, \mathrm{s}, \mathrm{H}-5{ }^{`}\right), 1.49(3 \mathrm{H}, \mathrm{s}, \mathrm{H}-$ 4), $2.32\left(3 \mathrm{H}, \mathrm{s}, 3-\mathrm{CH}_{3}\right), 3.95\left(3 \mathrm{H}, \mathrm{s}, 7-\mathrm{OCH}_{3}\right), 3.98$ $\left(3 \mathrm{H}, \mathrm{s}, 6-\mathrm{OCH}_{3}\right), 5.69\left(1 \mathrm{H}, \mathrm{d}, J=10.0, \mathrm{H}-2^{\prime}\right), 6.60$ $\left(1 \mathrm{H}, \mathrm{d}, J=10.0 \mathrm{~Hz}, \mathrm{H}-1^{\prime}\right), 6.93(1 \mathrm{H}, \mathrm{s}, \mathrm{H}-8), 7.38$ (1H, s, H-4), 7.55 (1H, s, H-5).

O-demethyl murrayanine (7): colourless mass; ${ }^{1} \mathrm{H}$ NMR (400 MHz, $\left.\mathrm{CDCl}_{3}\right): \delta 6.95(1 \mathrm{H}$, br t, H-6), $6.98(1 \mathrm{H}, \mathrm{dd}, 1-\mathrm{OH}), 7.47(1 \mathrm{H}, \mathrm{br} \mathrm{d}, J=8.0 \mathrm{~Hz}, \mathrm{H}-$ 7), $7.50(1 \mathrm{H}, \mathrm{d}, J=8.4 \mathrm{~Hz}, \mathrm{H}-2), 7.88(1 \mathrm{H}, \mathrm{d}, J=3.6$ Hz, H-8), 7.99 (1H, d, $J=8.5 \mathrm{~Hz}, \mathrm{H}-5), 8.30(1 \mathrm{H}, \mathrm{s}$, $\mathrm{NH}), 8.49(1 \mathrm{H}, \mathrm{s}, \mathrm{H}-4), 10.07$ (1H, s, 3-CHO).

\section{RESULTS AND DISCUSSION}

A total of seven compounds (1-7) were isolated from the methanol extract and its carbon tetrachloride and pet ether soluble fraction of leaves of $M$. koenigii by gel permeation chromatography over lipophilic Sephadex LH-20, preparative thin layer chromatography (PTLC) and high performance liquid chromatography (HPLC) on $\mathrm{C}_{18}$ bonded silica gel HPLC and repeated chromatographic separation by gel permeation chromatography. The structures of the isolated compounds were primarily solved by high field NMR data analysis.

The ${ }^{1} \mathrm{H}$ NMR (400 $\mathrm{MHz}, \mathrm{CDCl}_{3}$ ) spectrum of compound 1 revealed signals characteristic of a polycyclic acridone-type alkaloid, where two of the three hydroxyl groups were methylated $(\mathrm{OMe})$. The ${ }^{13} \mathrm{C}$ NMR $\left(100 \mathrm{MHz}, \mathrm{CDCl}_{3}\right)$ spectrum displayed a total of 16 carbon resonances, including an $\mathrm{N}-\mathrm{CH}_{3}$ signal at $\delta 34.2$ and a signal for carbonyl carbon at $180.8 \mathrm{ppm}$. The DEPT spectrum indicated that out of the 16 carbons, 8 had attached protons. It also demonstrated the presence of three methyls, five methines and eight quaternary carbon resonances.

The ${ }^{1} \mathrm{H}$ NMR spectrum exhibited signals for a highly characteristic $\mathrm{ABCD}$ spin system with four aromatic proton resonances at $\delta 7.50(1 \mathrm{H}, \mathrm{d}, J=8.5 \mathrm{~Hz}), 7.72$ $(1 \mathrm{H}, \mathrm{t}, J=8.5 \mathrm{~Hz}), 7.25(1 \mathrm{H}, \mathrm{t}, J=8.5 \mathrm{~Hz})$ and 8.42 $(1 \mathrm{H}$, br d, $J=8.5 \mathrm{~Hz})$, which could be assigned to four adjacent protons H-5, H-6, H-7 and H-8, respectively on ring A. A sharp singlet at $\delta 6.27$ that integrated for one proton was attributable to the aromatic proton at $\mathrm{C}-4$ of ring $\mathrm{C}$. Two sharp singlets, each of three proton intensity, at $\delta 3.92$ and 4.01 were ascribed to two methoxyl groups. The ${ }^{13} \mathrm{C}$ NMR 
spectrum also showed two methoxyl carbons resonating at $\delta 56.0$ and 60.8 . The high field value at $\delta 56.0$ was assigned to the sterically hindered methoxyl group at $\mathrm{C}-2$, while that at 60.8 could be attributed to C-3 methoxyl carbon. The signal at $\delta$ 180.8 clearly showed that the C-9 position had a ketonic functionality. On the other hand, the three proton singlet at $\delta 3.84$ could be assigned to the methyl on a nitrogen atom $\left(\mathrm{N}-\mathrm{CH}_{3}\right)$. This was substantiated by the ${ }^{13} \mathrm{C}$ resonance at $\delta 34.2$.<smiles>COc1cc2c(c(O)c1OC)c(=O)c1ccccc1n2C</smiles>

Arborinine (1)<smiles>COc1cc(/C=C\C(=O)O)ccc1O</smiles>

Ferulic acid (2)<smiles>O=c1ccc2ccc(O)cc2o1</smiles>

Umbelliferone (3)

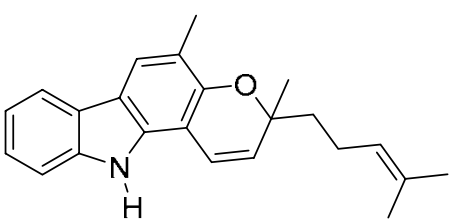

Mahanimbine (4)<smiles>COc1cc2[nH]c3c4c(c(C)cc3c2cc1OC)OC(C)(C)C=C4</smiles>

Koenimbine (5)<smiles>COc1cc2[nH]c3c4c(c(C)cc3c2cc1OC)OC(C)(C)C=C4</smiles>

Koenidine (6)<smiles>O=Cc1cc(O)c2[nH]c3ccccc3c2c1</smiles>

O-demethyl murrayanine (7)
The ${ }^{13} \mathrm{C}$ NMR spectrum $\left(150 \mathrm{MHz}, \mathrm{CDCl}_{3}\right.$ ) of compound 2 showed a total of 11 carbon resonances, including a carboxylic acid group at $\delta 167.4$ and a methoxyl group at 55.0. The ${ }^{1} \mathrm{H}$ NMR spectrum $\left(\mathrm{CDCl}_{3}, 600 \mathrm{MHz}\right)$ displayed a singlet of three proton intensity at $\delta 3.91$ suggesting the presence of a methoxyl gruop. It also exhibited two doublets centred at $\delta 7.60(1 \mathrm{H}, J=1.5 \mathrm{~Hz})$ and $6.95(1 \mathrm{H}, J=$ $8.4 \mathrm{~Hz})$ and a double doublet $(J=8.4,1.5 \mathrm{~Hz})$ at $\delta$ 7.08 typical for a 1,3,4-trisubstituted aromatic moiety. The doublets $(J=16.9 \mathrm{~Hz})$ centred at $\delta 7.60$ and 6.29 could be assigned to the trans coupled protons $\mathrm{H}-7$ and $\mathrm{H}-8$, respectively. The relatively low field resonance of $\mathrm{H}-7$ could be explained by its beta position to the carbonyl group, probably in the form of a carboxylic acid. Thus the structure of compound 2 was deduced as ferulic acid (2). The ${ }^{1} \mathrm{H}$ and ${ }^{13} \mathrm{C}$ NMR resonances in compound 2 were assigned by
2D NMR data notably HSQC and HMBC (Table 1) which allowed to revise the previous assignments made by Sajjadi et al.(2012). ${ }^{20}$ This is the first report of its isolation from M. koenigii.

Table 1. HMBC and HSQC correlations observed for Ferulic acid.

\begin{tabular}{|c|c|c|c|}
\hline \multirow{2}{*}{ Proton } & \multicolumn{2}{|r|}{ HMBC correlations } & \multirow[t]{2}{*}{ HSQC } \\
\hline & $2 \mathrm{~J}$ & $3 \mathrm{~J}$ & \\
\hline $\mathrm{H}-3$ & $\begin{array}{l}146.8 \\
(\mathrm{C}-2)\end{array}$ & $144.6(\mathrm{C}-7), 147.9(\mathrm{C}-1)$ & 109.3 \\
\hline $\mathrm{H}-5$ & & $109.3(\mathrm{C}-3), 144.6 \quad(\mathrm{C}-7)$ & 123.1 \\
\hline H-6 & & $\begin{array}{l}127.1(\mathrm{C}-4), 146.8 \\
(\mathrm{C}-2), 147.9(\mathrm{C}-1)\end{array}$ & 114.7 \\
\hline $\mathrm{H}-7$ & & $\begin{array}{l}109.3 \text { (C-3), } 115.8(\mathrm{C}-8), 123 \\
(\mathrm{C}-5), 127.1 \text { (C-4), } 167.4 \text { (C- } \\
9)\end{array}$ & 144.6 \\
\hline $\mathrm{H}-8$ & $\begin{array}{l}127.1 \\
(\mathrm{C}-6)\end{array}$ & $167.4(\mathrm{C}-9)$ & 115.8 \\
\hline $1-\mathrm{OH}$ & & $114.6(\mathrm{C}-6), 146.8(\mathrm{C}-2)$ & - \\
\hline 2-OMe & & $146.8(\mathrm{C}-2)$ & 55.9 \\
\hline
\end{tabular}


The compound $\mathbf{3}-\mathbf{7}$ were identified as umbelliferone $^{21}$, mahanimbine ${ }^{6}$, koenimbine ${ }^{5}$, koenidine $^{5}$ and $O$-demethyl murrayanine ${ }^{22}$, respectively through co-TLC with authentic samples, whenever possible and their structures were elucidated by comparison their spectral data with the published values reported previously.

\section{REFERENCES}

1. Biswas, A. 2006. Indigenous knowledge of herbal medicine and in vitro propagation of some rare medicinal plants in Chittagong Hill Tracts. PhD Dissertation. Rajshahi University, Rajshahi, Bangladesh.

2. Chowdhury, J.U., Bhuiyan, M.N.I. and Yusuf, M. 2008. Chemical components of the leaf essential oils of Murraya koenigii (L.) Spreng. and Murraya paniculata (L.) Jack. J. Bangladesh Pharmacol. Soc. 3, 59-63.

3. Handral, H.K., Pandith, A. and Shruthi, S.D. 2012. A review on Murraya Koenigii: multipotential medicinal plant. Asian J. Pharm.Clin. Res. 5, 5-14.

4. Sukari, M.A., Ahmad, K., Haron, M.J. and Muse, R. 2001. Carbazole alkaloids from roots of Murraya koenigii (Rutaceae). Malaysian J. Anal. Sci.7, 263-265

5. Shoeb, M., Hasan, Z., Saha, N.K., Karim, M.M. and Nahar, N. 2013. Antimicrobial activity of carbazole alkaloids from Murraya koenigii (L) Spreng. leave. Int. J. Med. Arom. Plants. 3, 131-135.

6. Nagappan, T., Ramasamy, P., Wahid, M.E.A., Segaran, T.C. and Vairappan, C.S. 2011. Biological acitivity of carbazole alkaloids and essential oil of Murraya koenigii against antibiotic resistant microbes and cancer cell lines. Molecules 16, 9651-9664.

7. Bakar, N.H.A., Sukari, M.A., Rahmani, M., Sharif, A.M., Khalid, K. and Yusuf, U.K. 2007. Chemical constituents from stem barks and roots of Murraya koenigii (Rutaceae). Malaysian J. Anal. Sci. 11, 173-176.

8. Bhattacharyya, P., Maiti, A.K., Basu, K. and Chowdhury, B.K. 1994. Carbazole alkaloids from Murraya koenigii. Phytochemistry 35, 1085-6.

9. Reisch, J., Goj, O., Wickramasinghe, A., Herath, H.M.T. and Henkel, G. 1992. Carbazole alkaloids from seeds of Murraya koenigii. Phytochemistry 31, 2877-9.

10. Nutan, M.T.H., Hasan, C.M. and Rashid, M.A. 1999. Bismurrayafoline E, a new dimeric carbazole alkaloid from Murraya koenigii. Fitoterapia LXX, 130-133.
11. Wong, K.C. and Tie, D.Y. 1993. The essential oil of the leaves of Murraya koenigii Spreng. J. Essent. Oil Res. 5, 371-374.

12. Reisch, J., Adebajo, A.C., Kumar, V. and Aladesanmi, A.J. 1994. Two carbazole alkaloids from Murraya koenigii. Phytochemsitry 36, 1073-1076.

13. Rajendran, B.T., Pallaiyan, B.B. and Selvaraj, N. 2014. Chemical composition, antibacterial and antioxidant profile of essential oil from Murraya koenigii (L.) leaves. Avicenna J. Phytomed. 4, 200-214.

14. Kaisar, M.A., Rahman, M.S., Rahman, M.Z., Hasan, C.M. and Rashid, M.A. 2011. A review on phytochemicals from some medicinal plants of Bangladesh. J. Phar. Nutri. Sci. 1, $87-95$.

15. Begum, F., Haque, M.R., Nahar, K.S. and Rashid, M.A. 2014. Secondary metabolites from different extractives of Stereospermum suaveolens. Dhaka Univ. J. Pharm. Sci. 13, 31-36.

16. Sikder, A.A., Sharmin, T., Rahman, A.F.M.M., Hasan, C.M. and Rashid, M.A. 2013. Screenings of four medicinal plants of Bangladesh for bioactivities. Dhaka Univ. J. Pharm. Sci. 12, 59-62.

17. 17. Ara, K., Haque, M.R., Kaiser, M.A., Rahman, A.H.M.M., Hasan, C.M. and Rashid, M.A. 2012. A new diarylheptanoid from Garuga pinnata Roxb. Dhaka Univ. J. Pharm. Sci. 12, 165-167.

18. Haque, M.R., Rahman, K.M., Begum, B., Hasan, C.M. and Rashid, M.A.2005. Secondary metabolites from Stereospermum chelonoides. Dhaka Univ. J. Pharm. Sci. 4, 61-64.

19. Rahmani, M., Muhammad, R. and Ali, A.M. 2010. Alkaloids and sulphur-containing amides from Glycosmis citrifolia and Glycosmis elongate. Sains Malaysiana 39, 445-451.

20. Sajjadi, S.E., Shokoohinia, Y. and Moayedi, N. Isolation and identification of ferulic acid from aerial parts of Kelussia odoratissima Mozaff. Jundishapur J. Nat. Pharm. Prod. 7, 159-162.

21. Kim, J.S., Kim, J.C., Shim, S.H., Lee, E.J., Jin, W.Y., Bae, K., Son, K.H., Kim, H.P., Kang, S.S. and Chang, H.W. 2006. Chemical constituents of the root of Dystaenia takeshimana and their anti inflammatory activity. Arch. Pharm. Res. 29, 617-623.

22. Ngadjui, B.T., Ayafor, J.F., Sondengam, B.L. and Connolly, J.D. 1989. Quinolone and carbazole alkaloids from Clausena anisata. Phytochemistry 28, 1517-1519.

23. Van Wagenen, B.C., Larsen, R., Cardellina, J.H., Randazzo, D., Lidert, Z.C. and Swithenbank, C. 1993. Ulosantoin, a potent insecticide from the sponge Ulosa ruetzleri. J. Org. Chem. 58, 335-337. 